Digitally-enabled diverse economies: exploring socially inclusive access to the circular economy in the city

Lekan, Małgorzata ${ }^{\text {a* }}$ and Rogers, Heather A. ${ }^{\mathrm{a}}$

${ }^{a}$ Department of Geography, Geology and Environment, Hull, UK

*M.Lekan@hull.ac.uk, +44 7821470000

This is an Accepted Manuscript of an article published by Taylor \& Francis in Urban geography on 21 Jul 2020, available online:

https://www.tandfonline.com/doi/full/10.1080/02723638.2020.1796097 


\title{
Digitally-enabled diverse economies: exploring socially inclusive access to the circular economy in the city
}

\author{
Abstract \\ Technology is increasingly reshaping social relations and transforming associated \\ urban spatial configurations. Advancements such as digital social platforms and \\ mobile apps have helped to connect social actors, empower citizens, and enable \\ diverse circular economy practices in cities. Paradoxically, technology is also \\ contributing to loneliness, anxiety, and depression among urban populations, and \\ raises nuanced questions about access and inclusivity in increasingly digitized \\ urban settings. In this contribution, we consider the possibilities and challenges \\ inherent in applying digital technologies to leverage the development of inclusive \\ and diverse circular economic spaces around a city's reuse, repair and recycling \\ infrastructure and drive socio-ecological transformations of urban spaces. We \\ conclude that uneven access to digital tools potentially reinforces existing urban \\ social inequalities, and that emphasis must be placed on understanding how \\ technologies should be more effectively designed and leveraged for a socially \\ inclusive circular economy in the city.
}

Keywords: circular economy; diverse economies; digital technologies; social inclusion; urban spaces

\section{Full Text}

By blurring the boundaries between the physical, digital, and socio-biological realms, technology has significant potential to reconfigure social relations and empower urban citizens through advancements such as Global Positioning Systems (GPS) and digital platforms (Warf, 2018). Whilst research on smart and interactive cities has raised questions about the social inclusiveness of such technologies (Nam \& Pardo, 2011), we know relatively little about the role of digital technologies in promoting socially inclusive and circular economy (CE)-based forms of urban economic development by diverse social actors and community-based organisations. Given that the social 
dimension of the $\mathrm{CE}$ concept is itself underrepresented in academic literatures (Hobson \& Lynch, 2016; Ranta, Aarikka-Stenroos, Ritala \& Mäkinen, 2018), we seek to prompt a debate over to what extent digital technologies deployed by various social actors enable or hinder inclusive access to citizen-led CE initiatives in cities within the developed world. We specifically focus on the city's reuse, repair and recycling infrastructure.

Our initial conceptual point of departure is the diverse economies literature, which seeks to render more visible in scholarship and social practice those diverse economic spaces and practices found in cities that represent an alternative to mainstream capitalist practices (Gibson-Graham, 2006). While diverse economies constitute an integral part of contemporary urban economic activity, and may embody both for-profit (capitalist) and not-for-profit characteristics, they are distinct from conventional economies insofar as their primary ambition is to generate value for people and the planet. Framing alternative CE practices in the diverse economies framework can therefore enrich CE literature, which has hitherto largely examined the inclusionary benefits and shortcomings of for-profit, market-led initiatives (Schor \& Attwood-Charles, 2017).

Diverse economic spaces and activities not only add to the city's vibrancy by supporting the local development of a closed-loop economy, but they are also increasingly mediated through, or completely dependent upon, digital tools such as social media platforms or mobile apps. Examples of such local economic spaces and practices - which we refer to as diverse circular economies (DCEs) - include reuse, repair and recycling infrastructure such as craft spaces, repair cafés, food sharing, local food production, litter picks, clothing swaps or makerspaces. In addition to boosting 
resource efficiency, DCEs foster social interactions and a sense of community belonging and inclusion in otherwise increasingly 'individualized' and divided urban societies (Slade, 2012). Technology can also act as a tool for social empowerment, especially for those urban actors seeking to address social and environmental challenges such as minimizing food waste, poverty, or the broader impacts of climate change in the city. Digital tools can promote urban social and environmental sustainability, as well as coordinate and connect like-minded individuals by offering access to diverse virtual spaces and mediating real life DCE activities and events (Berkhout \& Hertin, 2001; Pitt, Rychwalska, Roszczyńska-Kurasińska \& Nowak, 2019).

Concerning user engagement, it is widely acknowledged that digital platforms are capable of connecting individuals and facilitating knowledge exchange across ever-widening spatialities (Warf, 2018). Technology may to some degree, however, detract from the broader social benefits offered by DCEs outlined above. For example, overuse of technology has been shown to have negative social consequences, exacerbating loneliness and related urban mental health issues such as depression and anxiety (Slade, 2012; Thomée, Eklöf, Gustafsson, Nilsson \& Hagberg, 2007). Urban citizens may therefore be prevented from realizing their full social potential by limiting everyday social interactions and practices to virtual, anonymous, and purely transactional activities. In the context of the DCEs, these activities might include door-to-door delivery of local food or individual donations of unwanted goods to digitally mapped recycling points (e.g. Intellibins app). While in such instances urban social interactions can be limited, these activities nonetheless indirectly benefit wider urban society and the environment by supporting more efficient intra-urban logistics and local economic development. 
Urban digital platforms are often celebrated for enabling widespread access and removing barriers to social inclusion. On one hand, such platforms may be collectively owned (see Platform Cooperativism Consortium), which challenge the power dynamics embodied in traditional ownership structures. These alternative structures have implications for access and inclusion by potentially offering lower barriers to entry, participatory decision-making and adaptive capacity to address emerging community needs. Nonetheless, the access to these platforms or mobile apps, which increasingly underpin citizen participation in DCEs, requires access to Internet, ownership of, or access to, an electronic device, and capacity to maintain and utilize it. In a context where local resources are limited, certain groups, including low income or homeless individuals, may be excluded from engaging in DCE spaces (Humphry, 2014). On the other hand, many municipalities worldwide are increasingly offering free WiFi hotspots and universal access to required devices. This does not, however, solve the hidden problem of digital illiteracy, which is concentrated around the elderly and deprived, poorly educated urban communities (Datta, Bhatia, Noll \& Dixit, 2018), and can impede citizen participation in DCE activities, especially in cities with disproportionately higher concentrations of such social groups.

The increased reliance on technology in DCEs may also further reinforce existing urban inequalities such as disability and mental health. For example, Foley \& Ferri (2012) assert that "virtual worlds continue to privilege the able body by conforming to the social realities and norms of the non-virtual world" (p. 193). For example, many apps facilitating DCEs rely entirely on sight for participation, thereby reinforcing the predominant view that unimpaired vision is 'normal' and required for citizen engagement. Foley \& Ferri (2012) argue that inclusive technology must be 
universally designed, rather than additions made as accommodations for those not representing the culturally accepted constitution of 'normal'. Furthermore, Treviranus (2014) contends that socially inclusive technologies can better benefit wider urban society when they are designed for those with cognitive and/or physical disabilities, which are often hidden features of urban societies. For example, widely used text-to-speech technology was initially designed for disabled individuals. As DCE activities continue to rely on digital tools to function, socially inclusive design must be considered in order to prevent structural barriers to universal access in cities.

In summary, this discussion has explored the extent to which digital tools enable the effective mediation of DCE spaces, which in turn help citizen-groups to address the pressing socio-environmental challenges facing urban populations. We conclude that digital technologies can increase the visibility of DCEs and are important for understanding the spatial dynamics and deep structural problems underpinning contemporary urban life. While DCE activities may become more digitally visible, they may also remain hidden, inaccessible and unnavigable urban spaces for those without access to such platforms. Moreover, while the benefits of technology use in citizen-led DCE initiatives holds potential to foster increased urban connectivity, there remains a need to critically examine the social and environmental trade-offs associated with the daily urban use of technology. Particularly in reference to developing the social dimension of $\mathrm{CE}$, it is essential that universal accessibility in the design of technology is explored and leveraged to facilitate a more socially inclusive DCE in the city, and that the ownership structures of such technology are rendered transparent. 


\section{ORCID}

Małgorzata Lekan https://orcid.org/0000-0001-6299-335X

Heather Rogers https://orcid.org/0000-0003-2373-5010

\section{Acknowledgements}

This project has received funding from the European Union's Horizon 2020 research and innovation programme under the Marie Skłodowska-Curie grant agreement No 765198. For more information visit http://cresting.hull.ac.uk. 


\section{References}

1. Berkhout, Frans, \& Hertin, Julia (2001). Impacts of information and communication technologies on environmental sustainability: Speculations and evidence (Report No. 21). Brighton: OECD.

2. Datta, Arijit; Bhatia, Vimal, Noll, Josef, \& Dixit, Sudhir (2018). Bridging the digital divide: Challenges in opening the digital world to the elderly, poor, and digitally illiterate. IEEE Consumer Electronics Magazine, 8(1), 78-81.

3. Foley, Alan, \& Ferri, Beth A. (2012). Technology for people, not disabilities: ensuring access and inclusion. Journal of Research in Special Educational Needs, 12(4), 192-200.

4. Gibson-Graham, Julie Katherine (2006). A postcapitalist politics. Minneapolis: University of Minnesota Press.

5. Hobson, Kersty, \& Lynch, Nicholas (2016). Diversifying and de-growing the circular economy: Radical social transformation in a resource-scarce world. Futures, 82, 15-25.

6. Humphry, Justine (2014). The Importance of Circumstance: digital access and affordability for people experiencing homelessness. Australian Journal of Telecommunications and the Digital Economy, 2(3), 55.1-55.15.

7. Nam, Taewoo, \& Pardo, Theresa A. (2011). Conceptualizing smart city with dimensions of technology, people, and institutions, Proceedings of the 12th annual international digital government research conference: digital government innovation in challenging times, 282-291.

8. Pitt, Jeremy, Rychwalska, Agnieszka, Roszczyńska-Kurasińska, Magdalena, \& Nowak, Andrzej (2019). Democratizing Platforms for Social Coordination. IEEE Technology and Society Magazine, 38(1), 43-50.

9. Ranta, Valtteri, Aarikka-Stenroos, Leena, Ritala, Paavo, \& Mäkinen, Saku J. (2018). Exploring institutional drivers and barriers of the circular economy: a cross-regional comparison of China, the US, and Europe. Resources, Conservation and Recycling, 135, 70-82. 
10. Schor, Juliet B., Attwood-Charles, William (2017). The "sharing" economy: labor, inequality, and social connection on for-profit platforms. Sociology Compass, 11(1), e12493.

11. Slade, Giles (2012). The big disconnect: the story of technology and loneliness. New York: Prometheus Books.

12. Thomée, Sara, Eklöf, Mats, Gustafsson, Ewa, Nilsson, Ralph, \& Hagberg, Mats (2007). Prevalence of perceived stress, symptoms of depression and sleep disturbances in relation to information and communication technology (ICT) use among young adults - an explorative prospective study. Computers in Human Behavior, 23(3), 1300-1321.

13. Treviranus, Jutta (2014). Leveraging the web as a platform for economic inclusion. Behavioral sciences \& the law, 32(1), 94-103.

14. Warf, Barney (2018). Digital technologies and reconfiguration of urban space. In Ward, Kevin, Jonas, Andrew E. G., Miller, Byron, \& Wilson, David (Eds.), The Routledge Handbook on Spaces of Urban Politics (pp. 92-316). London: Routledge. 